\title{
Development of lymphoma from the donor of haploidentical stem cell transplantation: A case report
}

\author{
LINNA XIE and FANG ZHOU \\ Department of Hematology, The General Hospital of Jinan Military District, Jinan, Shandong 250031, P.R. China
}

Received June 4, 2016; Accepted August 31, 2017

DOI: $10.3892 / \mathrm{mco} .2017 .1414$

\begin{abstract}
Post-transplantation lymphoproliferative disease (PTLD) is a serious complication following hematopoietic stem cell transplantation (HSCT). The majority of the cases develop during the first year after the transplantation and are associated with reactivation of the Epstein-Barr virus (EBV); the EBV-induced lymphoproliferation usually includes donor-derived $B$ cells. We herein describe the case of a 28-year-old female patient who developed EBV-negative PTLD, namely diffuse large B-cell lymphoma (DLBCL), 6 months after receiving a haploidentical HSCT from her father. Chimerism analysis performed with XY fluorescence in situ hybridization revealed a B-cell PTLD originating from the donor. Unfortunately, the donor also developed DLBCL 380 days after donating progenitor cells, although he was hematologically normal at the time of donation. The present case demonstrated that disease transmitted from the donor may be a possible cause of PTLD.
\end{abstract}

\section{Introduction}

Post-transplantation lymphoproliferative disorder (PTLD) has been extensively described as a serious complication following allogeneic hematopoietic stem cell transplantation (HSCT) and solid-organ transplantation (SOT). The incidence of PTLD varies from 1 to $20 \%$, according to the type of organ transplant (1). It was recently reported that the incidence rate of monomorphic PTLD following allogeneic SCT was $0.41 \%$ (3/730). The time to development of PTLD in the 3 reported cases was 5.0, 5.8 and 5.9 months after receiving allogeneic $\operatorname{SCT}(2)$.

The majority of PTLD cases are associated with Epstein-Barr virus (EBV) infection. In the immunosuppressed state following transplantation, suppression of T-cell activity

Correspondence to: Dr Linna Xie, Department of Hematology, The General Hospital of Jinan Military District, 25 Shifan Road, Jinan, Shandong 250031, P.R. China

E-mail: xielinnadoctor@hotmail.com

Key words: post-transplantation lymphoproliferative disorder, lymphoma, haploidentical stem cell transplantation interferes with immune surveillance and allows proliferation of latently infected B lymphocytes; the proliferation of a malignant B-cell clone results in the development of lymphoma $(3,4)$. However, it was recently reported that 20-30\% of PTLD cases were EBV-negative. EBV-negative PTLDs occur later compared with EBV-positive cases, have a higher proportion of monomorphic PTLD, and exhibit a more aggressive clinical course $(5,6)$. The changes in the immunosuppressive regimens and new, unidentified infectious agents may be involved in the pathogenesis of EBV-negative PTLD (5).

We herein present the case of a recipient who developed monomorphic PTLD of donor origin 6 months after haploidentical HSCT (haplo-HSCT); the donor also developed the same type lymphoma 1 year after the donation.

\section{Case report}

A 28-year-old female patient was admitted to the General Hospital of Jinan Military District (Jinan, China) due to continuous high fever for 1 week. The patient's hematological data revealed pancytopenia. Based on the result of the bone marrow (BM) examination, the patient was diagnosed with refractory anemia with excess blasts accompanied by trilineage dysplasia, with $11.5 \%$ of myeloblasts. Immunophenotyping of the blasts revealed 55.7, 89.2 and 79.2\% of CD13-, CD33and CD34-positive cells, respectively. Cytogenetic screening of the blasts revealed no chromosomal abnormalities. The patient suffered from repeated infectious episodes, such as upper respiratory tract infection and bacterial sepsis, and required blood transfusion. Due to the patient's poor general condition and considering that further chemotherapy may render her unsuitable for transplantation, she received transplantation without chemotherapy 3 months after admission. The conditioning regimen consisted of busulfan, cyclophosphamide, cytarabine and semustine. Short-term methotrexate, mycophenolate mofetil (MMF), cyclosporin A (CSA) and antithymocyte globulin (ATG) were used as graft-versus-host disease (GVHD) prophylaxis. The patient was an only child and did not have a suitable unrelated donor; thus, she received a haplo-HSCT from her father (aged 45 years) on February 1, 2013, with 5/6 human leukocyte antigen (HLA) locus matching. The recipient received mixed allografts of recombinant human granulocyte colony-stimulating factor (G-CSF; $5 \mathrm{mg} / \mathrm{kg} /$ day for 5 days)-mobilized bone marrow and peripheral blood stem cell harvests. EBV serological testing of the patient and the 
donor prior to transplantation was negative. The total infused dose of nucleated cells was $7.9 \times 10^{8} / \mathrm{kg}$ body weight, including $2.1 \times 10^{6} / \mathrm{kg} \mathrm{CD} 34-$ positive cells. If the neutrophil count was $<0.5 \times 10^{9} / 1,5 \mathrm{mg} / \mathrm{kg} /$ day of G-CSF were administered to help the recipient recover from granulocyte depletion. When the neutrophil count reached $>1.5 \times 10^{9} / 1$, the dose of G-CSF was slowly tapered. Neutrophil $\left(0.5 \times 10^{9} / 1\right)$ and platelet $\left(20 \times 10^{9} / 1\right)$ engraftment were observed on days 15 and 35, respectively, after the transplantation. Bone marrow examination on day 30 revealed a normocellular marrow without myelodysplasia. Cytogenetic analysis revealed a normal male karyotype of 46, XY. Fluorescence in situ hybridization (FISH) of the lymphocytes was also used for chimerism detection, which indicated $98 \%$ donor implant. At the 2-, 3-, 6-, 9- and 12-month follow-up, the chimerism was complete (100\%). On day 181 , the patient developed a skin rash, liver dysfunction and diarrhea, and was diagnosed with grade II GVHD. The GVHD gradually improved with prednisolone treatment. Bacterial pneumonia and hemorrhagic cystitis also occurred, but were controlled with extended-spectrum beta-lactamases (imipenem), and supportive treatment with hydration, diuretics and platelets, respectively.

Once the acute GVHD of the recipient was controlled, the immunosuppressive drugs were tapered. Six months after haplo-HSCT, a neoplastic lesion sized $1 \times 1.5 \mathrm{~cm}^{2}$ appeared on the recipient's head (frontotemporal region). The mass had a smooth surface, hard consistency, and was painless. Biopsy of the lesion was performed and the histopathological analysis revealed destruction of the normal structure, with diffuse invasion by cells exhibiting atypical nuclear bodies. The immunostaining for CD20, paired Box (PAX)-5 and B-cell lymphoma $(\mathrm{Bcl})-6$ was positive and the $\mathrm{Ki}-67$ index was 90\%, while cytomegalovirus (CMV) and EBV-encoded small RNA (EBER) were negative. A clonality assay by immunoglobulin heavy $(\mathrm{IgH})$ chain gene rearrangement study was also performed by polymerase chain reaction. The DNA extracted from the pathological tissues confirmed the monoclonal origin of the neoplasm. FISH study of a specimen from the patient confirmed the presence of XY male donor cells and revealed that the lymphoma cells were of donor origin (Fig. 1). The PTLD involved monomorphic B cells and the type was diffuse large B-cell lymphoma (DLBCL). Positron emission tomography-computed tomography (PET-CT) examination revealed hypermetabolic lesions in multiple lymph nodes, muscle, right breast and bone. PTLD was diagnosed on August 16,2013 , based on the abovementioned results. The patient was managed by withdrawal of the immunosuppressant drugs (CSA and MMF) and administration of chemotherapy, including two courses of rituximab, cyclophosphamide, doxorubicin, vincristine and prednisone (R-CHOP) and 4 courses of R-COP. PET examination showed remission and the patient achieved a significant relief of the signs and symptoms; she remained free of PTLD at the last follow-up in January 2016, 25 months after the diagnosis of the disease.

Although the donor was disease-free prior to the haplo-SCT, he was admitted to our hospital on February 22, 2014 (380 days after stem cell donation) due to left eye pain, blurred vision and fever for 3 days. The head magnetic resonance imaging examination revealed multiple lesions on the skull. On PET-CT, hypermetabolic lesions were identified in the prostate, left side of the seminal vesicle, multiple lymph nodes of the pelvic cavity and bone. There were no obvious symptoms of frequent micturition, urgent urination, urinary pain or hematuria. The free prostate-specific antigen (PSA) and total PSA levels were normal. The patient underwent transrectal prostate biopsy and the histopathological analysis revealed DLBCL. The immunostaining for CD20, PAX-5 and Bcl- 6 was positive, the Ki-67 index was $61 \%$, whereas CMV and EBER were negative. The lactate dehydrogenase level was $330 \mathrm{U} / 1$ and $\beta 2$-microglobulin level was $2.8 \mathrm{mg} / \mathrm{l}$. The donor was diagnosed with stage IVB DLBCL; he received 6 courses of R-CHOP chemotherapy and attained CR on August 13, 2014. He remained disease-free for 23 months until relapse in July, 2015; after 4 courses of R-CHOP chemotherapy, the patient achieved a second CR, and then he received an autologous stem cell transplantation in February, 2016. The conditioning treatment consisted of carmustine, etoposide, cytarabine and melphalan (BEAM regimen).

\section{Discussion}

PTLD is a life-threatening complication following SOT and allogeneic HSCT (allo-HSCT), which encompasses a heterogeneous group of lymphoproliferative disorders ranging from reactive, polyclonal hyperplasia to aggressive non-Hodgkin's lymphomas. The incidence of PTLD after allo-HSCT is $\sim 1 \%$, and the majority of cases occur PTLD within the first year of transplantation. The risk factors of PTLD include immunodeficiencies caused by high-dose chemotherapy and/or irradiation, T-cell depleted donor cells, the use of HLA-mismatched transplants, intensive immunosuppression with $\mathrm{T}$ cells to prevent GVHD, and antibodies, which may increase the risk for PTLD to up to $24 \%$ (7).

It was previously demonstrated that $60-70 \%$ of PTLD cases are associated with EBV infection (8). The presence of EBV in lymphoid proliferation in this setting has been used to support the diagnosis of PTLD $(9,10)$, determine clonal proliferation (11), and possibly identify patients at high risk of developing PTLD (12). Although a number of groups recommend EBV positivity to be a biomarker for diagnosing PTLD, several others have reported EBV-negative PTLD cases $(5,13)$. Compared with EBV-PTLD, the pathogenesis of EBV-negative PTLD is less well defined. EBV-negative PTLD tends to occur later compared with EBV-positive cases following transplantation, the majority of EBV-negative PTLD cases are of monomorphic type, more aggressive and carry an overall poorer prognosis (6). In all published reports, the average occurrence time of initial EBV-negative PTLD was at 50 months post-transplantation compared with 10 months for EBV-positive cases. It has been hypothesized that EBV-negative PTLD cases are associated with other viral infections, such as human herpes virus 8 and CMV $(14,15)$, and hit-and-run infection (16) or chronic antigen stimulation by the graft (17).

In the present case, both the patient and donor were EBV-negative, the patient suffered from monomorphic PTLD only 6 months after the transplantation, and the donor developed the same type of lymphoma 1 year after the donation. The interval to EBV-negative PTLD occurrence was significantly shorter compared with that previously reported (18). 

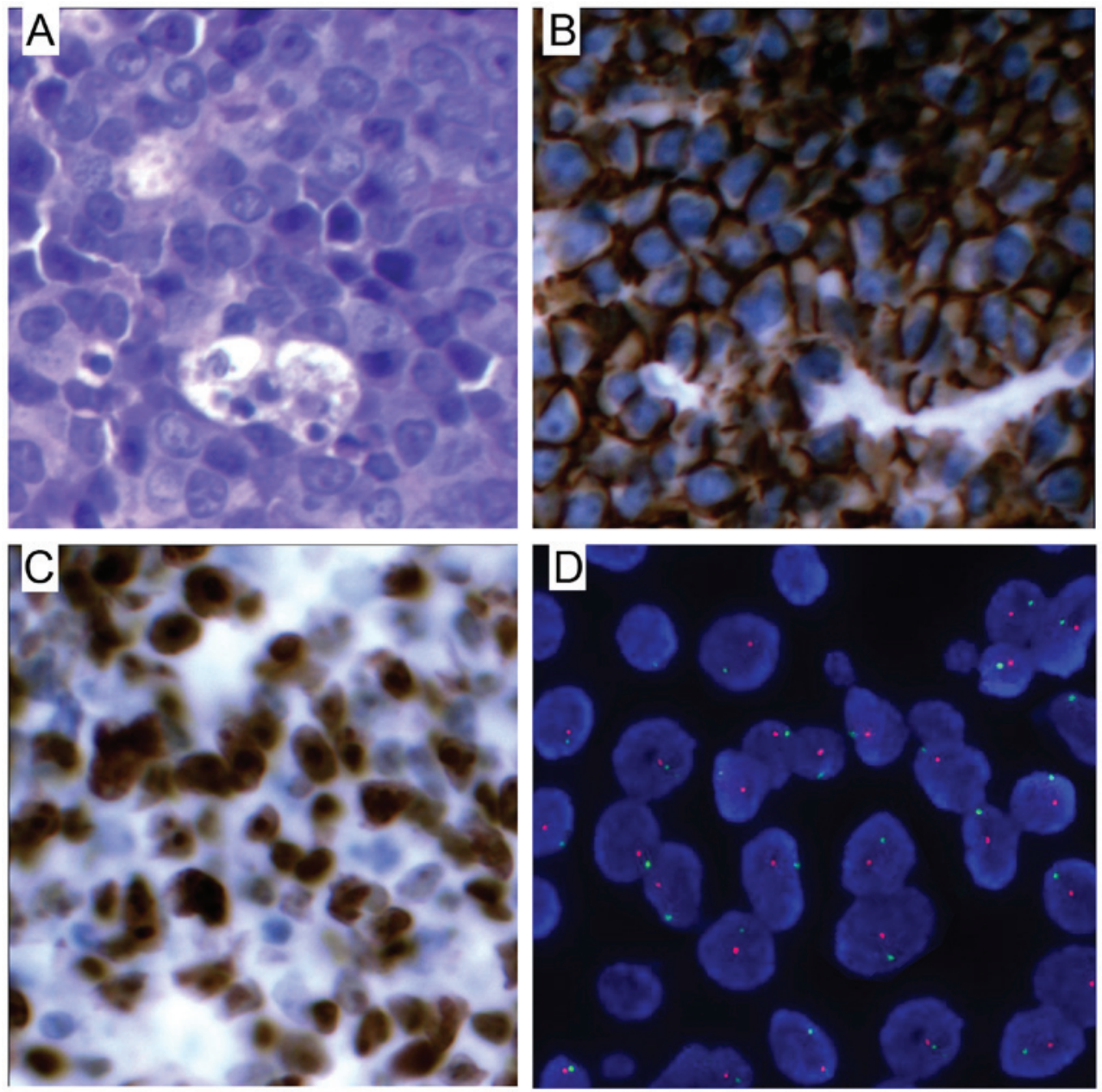

Figure 1. (A) Hematoxylin and eosin staining of biopsy sections from the neoplasm revealed lymphoma cell infiltration (magnification, $\mathrm{x} 40$ ). (B) Positive CD20 staining in the lymphoma cells (original objective magnification, x40). (C) Proliferative state of lymphoma cells by Ki-67 staining. (D) Fluorescence in situ hybridization of the X (green signal) and Y (red signal) chromosomes, showing male tumor cells in the lymphoma tissue.

The immunodeficiency caused by high-dose chemotherapy as part of the conditioning regimen, ATG, CSA and MMF to deplete donor T cells and prevent GVHD, haploidentical HSCT and acute GVHD were the important factors for PTLD. With the exception of the abovementioned reasons, preconditioning therapy, chemotherapy-induced recipient stromal abnormalities and transfection of a leukaemogenic agent (viral or non-viral) from host to donor cells may also be associated with malignant transformation of donor progenitor cells (19). Since the donor developed the same type of lymphoma 1 year after the donation, it is possible that the patient's PTLD was transmitted from donor to recipient. It was difficult to distinguish between donor-transmitted and donor-derived tumors.

Donor-related malignancies are frequently reported in solid organ transplants. According to a report from transplant centers, a total of 21 donor-related malignancies from 14 cadaveric and 3 living donors were reported in a cohort of 34,933 cadaveric donors and 108,062 recipients (20). A total of 15 malignancies were donor-transmitted and 6 were donor-derived. Transmitted tumors were defined as malignancies that are present in the donors at the time of transplantation, while derived tumors are de novo tumors that develop in transplanted donor hematogenous or lymphoid cells after the transplantation (20). In the present case, the donor was diagnosed with DLCBL 1 year after the donation. Although the physical and serological examination did not reveal any evidence of DLCBL, it cannot be excluded that the donor was in the period of transition from reactive or polyclonal hyperplasia to aggressive non-Hodgkin lymphoma at the time of the transplantation.

This case is noteworthy, as it demonstrates that lymphoma may be transmitted from the donor to the recipient by allo-HSCT, which is new evidence for the occurrence of PTLD. Furthermore, the donor was healthy prior to the donation. Although there is no direct evidence for the association between HSCT and the occurrence of lymphoma in the donor and the recipient, it should be noted that the health of the donor is a crucial factor.

In summary, we herein reported a case of PTLD transmitted from donor to recipient during HSCT. Thus, more sensitive screening assays should be performed to exclude donors with malignant hematological cancers.

\section{Acknowledgements}

The present study was supported by grants from the Natural Science Foundation of China (no. 8150010892) and the Presidential Foundation of the General Hospital of Jinan Military District (no. 2014ZD03). 


\section{References}

1. Lactase AS: Post-transplant lymphoproliferative disorders. Oncologist 11: 674-680, 2006.

2. Choi JH, Park BB, Suh C, Won JH, Lee WS and Shin HJ: Clinical characteristics of monomorphic post-transplant lymphoproliferative disorders. J Korean Med Sci 25: 523-526, 2010.

3. Savoie A, Perpête C, Carpentier L, Joncas J and Alfieri C: Direct correlation between the load of Epstein-Barr virus-infected lymphocytes in the peripheral blood of pediatric transplant patients and risk of lymphoproliferative disease. Blood 83 : 2715-2722, 1994.

4. Chang MS and Kim WH: Epstein-Barr virus in human malignancy: A special reference to Epstein-Barr virus associated gastric carcinoma. Cancer Res Treat 37: 257-267, 2005.

5. Leblond V, Davi F, Charlotte F, Dorent R, Bitker MO, Sutton L, Gandjbakhch I, Binet JL and Raphael M: Post-transplant lymphoproliferative disorders not associated with Epstein-Barr virus: A distinct entity? J Clin Oncol 16: 2052-2059, 1998.

6. Nelson BP, Nalesnik MA, Bahler DW, Locker J, Fung JJ and Swerdlow SH: Epstein-Barr virus-negative post-transplant lymphoproliferative disorders: A distinct entity? Am J Surg Pathol 24: 375-385, 2000.

7. Gottschalk S, Rooney CM and Heslop HE: Post-transplant lymphoproliferative disorders. Annu Rev Med 56: 29-44, 2005.

8. Loren AW, Porter DL, Stadtmauer EA and Tsai DE: Post-transplant lymphoproliferative disorder: A review. Bone Marrow Transplant 31: 145-155, 2003.

9. Frizzera G, Hanto DW, Gajl-Peczalska KJ, Rosai J, McKenna RW, Sibley RK, Holahan KP and Lindquist LL: Polymorphic diffuse B-cell hyperplasias and lymphomas in renal transplant recipients. Cancer Res 41: 4262-4279, 1981.

10. Hanto DW, Frizzera G, Purtillo DT, Sakamoto K, Sullivan JL, Saemundsen AK, Klein G, Simmons RL and Najarian JS: Clinical spectrum of lymphoproliferative disorders in renal transplant recipients and evidence for the role of Epstein-Barr virus. Cancer Res 41: 4253-4261, 1981.

11. Locker J and Nalesnik M: Molecular genetic analysis of lymphoid tumors arising after organ transplantation. Am J Pathol 135: 977-987, 1989.
12. Randhawa PS, Jaffe R, Demetris AJ, Nalesnik M, Starzl TE, Chen YY and Weiss LM: Expression of Epstein-Barr virus-encoded small RNA (by the EBER-1 gene) in liver specimens from transplant recipients with post-transplantation lymphoproliferative disease. N Engl J Med 327: 1710-1714, 1992.

13. Knowles DM, Cesarman E, Chadburn A, Frizzera G, Chen J, Rose EA and Michler RE: Correlative morphologic and molecular genetic analysis demonstrates three distinct categories of post-transplantation lymphoproliferative disorders. Blood 85: 552-565, 1995.

14. Capello D and Gaidano G: Post-transplant lymphoproliferative disorders: Role of viral infection, genetic lesions and antigen stimulation in the pathogenesis of the disease. Mediterr J Hematol Infect Dis 1: e2009018, 2009.

15. Mañez R, Breinig MC, Linden P, Wilson J, Torre-Cisneros J, Kusne S, Dummer S and Ho M: Posttransplant lymphoproliferative disease in primary Epstein-Barr virus infection after liver transplantation: The role of cytomegalovirus disease. J Infect Dis 176: 1462-1467, 1997.

16. Jox A, Rohen C, Belge G, Bartnitzke S, Pawlita M, Diehl V, Bullerdiek $J$ and Wolf $J$ : Integration of Epstein-Barr virus in Burkitt's lymphoma cells leads to a region of enhanced chromosome instability. Ann Oncol 8 (Suppl 2): S131-S135, 1997.

17. Morscio J, Dierickx D, Ferreiro JF, Herreman A, Van Loo P, Bittoun E, Verhoef G, Matthys P, Cools J, Wlodarska I, et al: Gene expression profiling reveals clear differences between EBV-positive and EBV-negative posttransplant lymphoproliferative disorders. Am J Transplant 13: 1305-1316, 2013.

18. Evens AM, David KA, Helenowski I, Nelson B, Kaufman D, Kircher SM, Gimelfarb A, Hattersley E, Mauro LA, Jovanovic B, et al: Multicenter analysis of 80 solid organ transplantation recipients with post-transplantation lymphoproliferative disease: Outcomes and prognostic factors in the modern era. J Clin Oncol 28: 1038-1046, 2010.

19. Brunstein CG, Hirsch BA, Hammerschmidt D, McGlennen RC, Nguyen PL and Verfaillie CM: Leukemia in donor cells after allogeneic hematopoietic stem cell transplant. Bone Marrow Transplant 29: 999-1003, 2002.

20. Myron Kauffman H, McBride MA, Cherikh WS, Spain PC, Marks WH and Roza AM: Transplant tumor registry: Donor related malignancies. Transplantation 74: 358-362, 2002. 